\title{
Studies on Some Benzoxazine-4-one Derivatives with Potential Biological Activity
}

\author{
Osman M. O. Habib, Hussein M. Hassan, Ahmed El-Mekabaty ${ }^{*}$
}

Department of Chemistry, Faculty of Science, Mansoura University, Mansoura, 35516, Egypt

\begin{abstract}
Toluenesulphonyloxy phenyl)-3,1-benzoxazine-4-one 2 was prepared and reacted with some nitrogen nucleophiles, e.g., ammonia, o-phenylendiamine, some heterocyclic amines, hydrazine hydrate and hydroxylamine hydrochloride and sulphur nucleophile, e.g., phosphorous pentasulphide. Structures of the newly synthesized compounds were established by elemental analysis and spectral data. All new prepared compounds were subjected to antimicrobial activity evaluation where compounds, 8, 9, 10 and 18 exhibited good activities against Bacillus Thuringenesis and 5, 10, 11, 14, 15, 17 and 18 exhibited good activities against Klebseilla Pneumonia. On the other hand, the results for antifungal activities revealed that, compounds 5, 6, 7, 16 and 17 exhibited good activities against Trichoderma Herzianum and Trichoderma Virdi.
\end{abstract}

Keywords Quinazolinone, Tetrazole, Thiazolidinone, Imidazole, Antibacterial

\section{Introduction}

Substituted benzoxazinone and quinazolinone derivatives have become of great importance due to their wide range of biological activity. Previous studies have reported that, they exhibit antitubercular, antihypertensive, anticancer, antiHIV, antiviral, anti-inflammatory, and antifungal activities[1,2]. Besides, they were used as analgesics, inhibitors for cathepsin G, Human leukocyte elastase, dual selective serotonin reuptake, and potent inactivators of $\mathrm{C}_{\mathrm{Ir}}$ serine protease[3]. On the other hand, it has been stated that compounds containing aromatic sulfonate or sulfonamide moieties possess high acaricidal as well as insecticidal activity[4].

\section{Results and Discussions}

As a part of our interest on the synthesis of biologically active molecules, the present investigation aims to synthesize a series of products bearing both aryl sulfonate, benzoxazinone and quinazolone moieties in the same molecule hoping that, these new products might show high biocide activity. Thus, 2-(4-toluene sulphonyloxy phenyl) -3,1benzoxazine-4-one 2 was prepared by treatment of one mole of anthranilic acid with two moles of the acid chloride $\mathbf{1}$ in the presence of dry pyridine (Scheme 1)[5].

Fusion of compound $\mathbf{2}$ with $o$-phenylenediamine in the presence of freshly fused sodium acetate at $100^{\circ} \mathrm{C}$ and $180^{\circ} \mathrm{C}$

* Corresponding author:

a_el_m11@yahoo.com (Ahmed El-Mekabaty)

Published online at http://journal.sapub.org/ajoc

Copyright (C) 2012 Scientific \& Academic Publishing. All Rights Reserved gives different products. When fusion was carried out at $100^{\circ} \mathrm{C}$, 4-(3-(2-aminophenyl)-4-oxo-3,4-dihydro quinazolin-2-yl) phenyl-4-methyl benzene sulfonate 4 was obtained. On the other hand, fusion of 2 at $180^{\circ} \mathrm{C}$ leads to the formation of 4-(4-benzo[4,5]imidazo[1,2-c] quinazolin -6-yl) phenyl- 4-methylbenzene sulfonate 3. Moreover, reaction of benzoxazinone $\mathbf{2}$ with $o$-phenylenediamine in absolute ethanol under reflux afforded 4-[2-[(N-2- aminophenyl) benzoyl] carbamoyl]phenyl-4'-methyl benzenesulfonate 5. Furthermore, fusion of $\mathbf{2}$ with ammonium acetate at $160-170^{\circ} \mathrm{C}$ afforded 4-(4-oxo-3,4-dihydroquinazolin -2-yl) phenyl-4'-methylbenzenesulfonate 6 (Scheme 2). The infra-red spectrum of 6 revealed stretching frequencies at 3336, $3173, \mathrm{~cm}^{-1}$ characteristic for the $-\mathrm{OH}$ and $-\mathrm{NH}$, groups, respectively. This illustrates that quinazolone derivative 6 exists in a lactam- lactim tautomeric equilibrium.

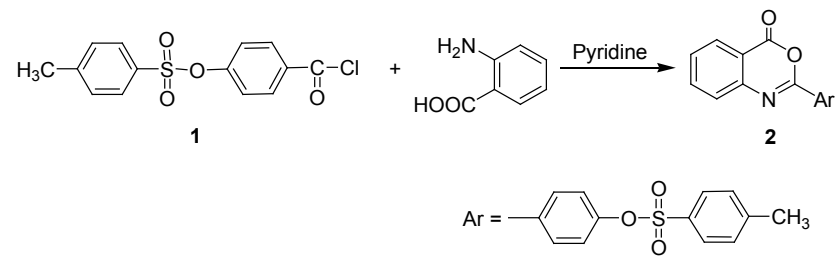

Scheme 1. Synthesis of 4-(4-oxo-4H-benzo[ $d][1,3]$ oxazin-2-yl)phenyl 4-methylbenzene-sulfonate (2)

On the other hand, compound 2 reacted with some heterocyclic amines, e.g., 4-aminoantipyrine, 2-aminopyridine and 3-aminopyridine afforded 7, 8 and 9 respectively. Moreover, benzoxazinone $\mathbf{2}$ reacts with phosphorous pentasulfide in dry xylene under reflux affording 4-(4-thioxo4H-3,1-benzothiazin-2-yl)phenyl-4-methylbenzenesulfonate 10 (Scheme 3). With the aim of expanding the synthetic potential of the quinazolones formed, we also studied the 
reaction of benzoxazinone 2 with both hydroxylamine hydrochloride and hydrazine hydrate. Thus, the reaction of $\mathbf{2}$ with hydroxylamine hydrochloride in ethanol in the presence of sodium acetate gives 4-(3-hydroxy-4-oxo-3,4- dihydroquinazolin-2-yl)phenyl-4-methyl benzene sulfonate 11. On the other hand, reaction of $\mathbf{2}$ with hydrazine hydrate in ethanol revealed 4-(3-amino-4-oxo-3,4- dihydriquinazolin-2-yl)phenyl-4-methyl benzene sulfonate 12 (Scheme 3).

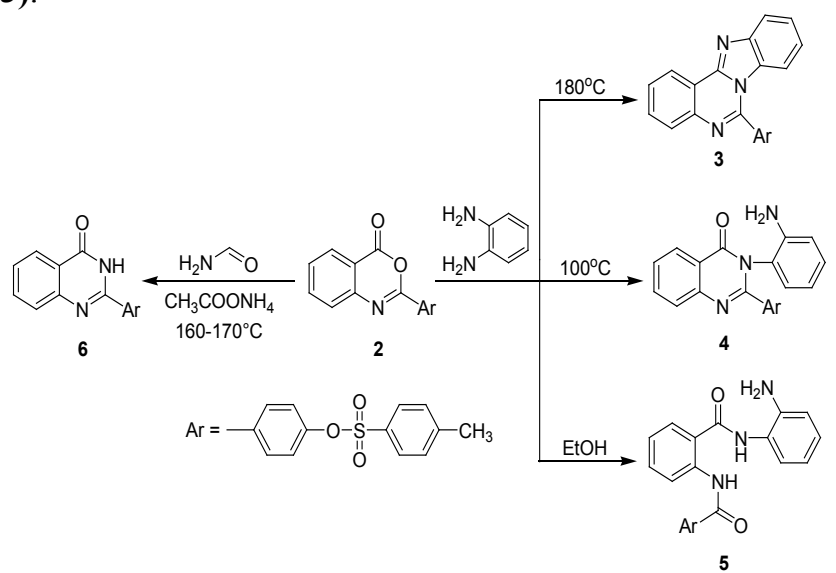

Scheme 2. Reaction of benzoxazinone 2 with $o$-phenylenediamine and formamide

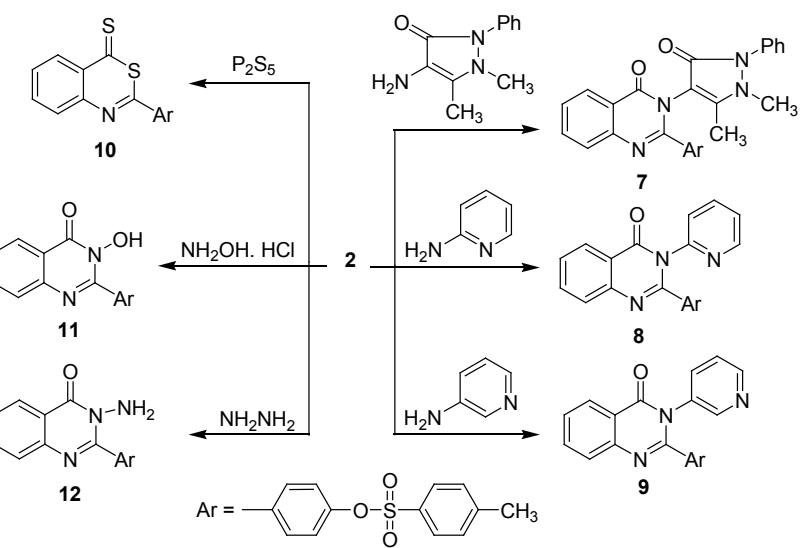

Scheme 3. Condensation of benzoxazinone 2 with different amines and phosphorus pentasulphide

Condensation of compound $\mathbf{1 2}$ with benzaldehyde in boiling ethanol containing few drops of piperidine afforded the corresponding 4-(3-(benzylideneamino)-4-oxo-3,4dihydroquinazolin-2-yl)phenyl-4-methylbenzenesulfonate 13. It was thus of interest in the present work to prepare some new products containing in their skeleton both the azetidinone and thiazolidinone moieties linked with quinazolone derivatives. Thus reaction of $\mathbf{1 3}$ with both chloroacetyl chloride and thioglycolic acid gave $\mathbf{1 4}$ and $\mathbf{1 5}$, respectively. Moreover, acetylation and benzoylation of $\mathbf{1 2}$ with acetylchloride and/or benzoylchloride afforded 4-(3,3-diacetamido-4-oxo-3,4-dihydroquinazolin-2-yl)phenyl-4-meth ylbenzenesulfonate 16 and 4-(4-oxo-3,3-(dibenzamido)-3, 4-dihydroquinazolin-2-yl)phenyl-4-methyl benzene sulfonate 17, respectively. Furthermore, when 3- aminoquinazolinone 12 condensed with phthalic anhydride by fusion in an oil bath at $160^{\circ} \mathrm{C}$ gives 4-(3-(1,3-dioxoisoindolin-2-yl)4-oxo-3, 4-dihydroquinazolin-2-yl) phenyl-4- methyl benzene sulfonate 18 (Scheme 4).

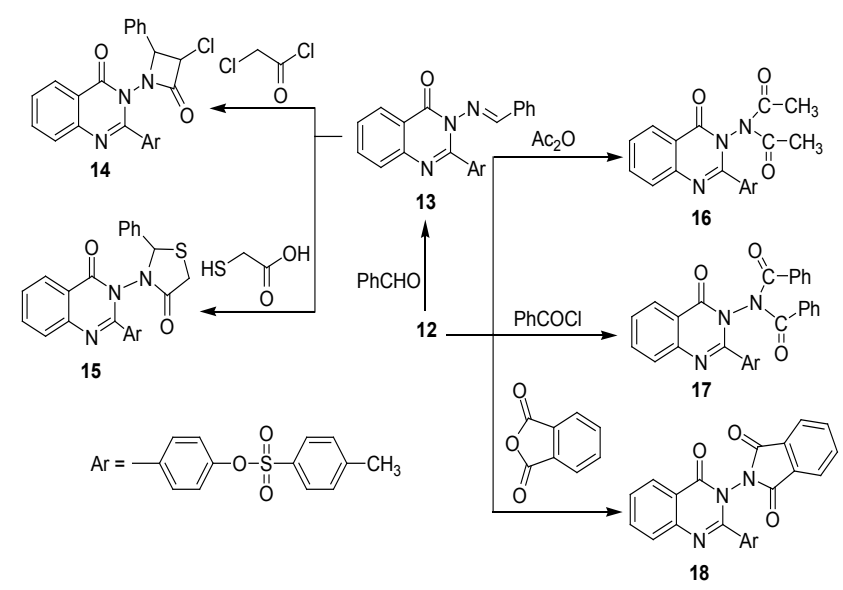

Scheme 4. Reactions of 3-aminoquinazolone derivative $\mathbf{1 2}$

\section{Antimicrobial Activity}

Most of the compounds showed very good antibacterial and antifungal activities and were almost competitive with the standard drugs. The results are summarized in Table 1, 2 .

The results for antibacterial activities depicted in Table 1 revealed that, compounds $3,5,7,8,9,10,11,17$ and 18 exhibited good activities against the reference chemotherapeutics due to the presence of antipyrine, pyridine and phthalimide moieties which play an important as antibacterial and antifungal, while 8, 9, 10 and 18 exhibited good activities against Bacillus Thuringenesis and compounds 5, 10, 11, 14, 15, 17 and 18 exhibited good activities against Klebseilla Pneumonia. On the other hand, the results for antifungal activities depicted in Table 2 revealed that, compounds 5, 6, 7, 16 and 17 exhibited good activities against Trichoderma Herzianum and Trichoderma Virdi.

A comparison of antibacterial and antifungal activities of compounds with their structures revealed that, the compounds that bearing aryl sulfonate, benzoxazinone and quinazolone moieties in the same molecule caused significant activity against Bacillus Thuringenesis, Klebseilla Pneumonia, Trichoderma Herzianum and Trichoderma Virdi.

\section{Experimental}

All melting points are recorded on Gallenkamp electric melting point apparatus. The IR spectra $v \mathrm{~cm}^{-1}(\mathrm{KBr})$ were recorded on Perkin ElmerInfrared Spectrophotometer Model 157, Grating. The ${ }^{1} \mathrm{H}$ NMR spectra were obtained on a Varian Spectrophotometer at $500 \mathrm{MHz}$ using TMS as an internal reference and DMSO-d6 as solvent. The mass spectra (EI) were recorded on $70 \mathrm{eV}$ with Kratos MS equipment and/or a Varian MAT 311 A Spectrometer. Elemental analyses were carried out at the Micro Analytical Center of Cairo Univ., Giza, Egypt. 
Table 1. Diameter of inhibited zones (I. Z. D.) in millimeters as a criterion of antibacterial activity of the synthesized compounds

\begin{tabular}{|c|c|c|c|c|c|}
\hline \multirow{3}{*}{ Comp. No. } & \multicolumn{2}{|c|}{ Bacteria } & \multirow{3}{*}{ Comp No. } & \multicolumn{2}{|c|}{ Bacteria } \\
\hline & B. Thuringenesis & K. Pneumonia & & B. Thuringenesis & K. Pneumonia \\
\hline & I. Z.D. $\mathrm{mm}$ & I. Z.D. $\mathrm{mm}$ & & I. Z.D. $\mathrm{mm}$ & I. Z.D. $\mathrm{mm}$ \\
\hline 2 & 27 & 33 & 12 & 27 & 35 \\
\hline 3 & 31 & 36 & 13 & 30 & 26 \\
\hline 4 & 27 & 30 & 14 & 29 & 45 \\
\hline 5 & 35 & 44 & 15 & 29 & 45 \\
\hline 6 & 29 & 33 & 16 & 32 & 39 \\
\hline 7 & 34 & 37 & 17 & 35 & 45 \\
\hline 8 & 39 & 38 & 18 & 39 & 50 \\
\hline 9 & 38 & 32 & Flummox & 24 & 29 \\
\hline 10 & 46 & 49 & Ampicillin & 29 & 34 \\
\hline 11 & 29 & 44 & Chloramphenicol & 19 & 28 \\
\hline
\end{tabular}

Table 2. Diameter of inhibited zones (I. Z. D.) in millimeters as a criterion of antifungal activity of the synthesized compounds at a concentration of 10 $\mathrm{mg} / \mathrm{ml}$

\begin{tabular}{|c|c|c|c|c|c|}
\hline \multirow{3}{*}{ Comp No. } & \multicolumn{2}{|c|}{ Fungi } & \multirow{3}{*}{ Comp No. } & \multicolumn{2}{|c|}{ Fungi } \\
\hline & T. Herzianum & T. Virdi & & T. Herzianum & T. Virdi \\
\hline & I. Z.D. $\mathrm{mm}$ & I. Z.D. $\mathrm{mm}$ & & I. Z.D. $\mathrm{mm}$ & I. Z.D. mm \\
\hline 2 & 35 & 39 & 12 & 31 & 48 \\
\hline 3 & 41 & 31 & 13 & 35 & 26 \\
\hline 4 & 37 & 32 & 14 & 33 & 39 \\
\hline 5 & 44 & 36 & 15 & 31 & 36 \\
\hline 6 & 49 & 40 & 16 & 48 & 49 \\
\hline 7 & 46 & 44 & 17 & 46 & 41 \\
\hline 8 & 40 & 48 & 18 & 40 & 46 \\
\hline 9 & 41 & 45 & Flummox & 35 & 41 \\
\hline 10 & 38 & 46 & Ampicillin & 41 & 36 \\
\hline 11 & 32 & 49 & Chloramphenicol & 32 & 31 \\
\hline
\end{tabular}

Synthesis of 2-(4-toluenesulphonyloxyphenyl)-3,1- Benzoxazine- 4-one (2)

To a solution of anthranilic acid (1.371 g, $0.01 \mathrm{~mole})$ in dry pyridine $(30 \mathrm{~mL})$, the acid chloride 1 (6.21 g, 0.02 mole) was added portion wise with stirring at room temperature. The reaction mixture was poured onto cold water $(100 \mathrm{~mL})$ and the precipitated solid was filtered off, washed with cold water, dried and recrystallized from ethanol to give benzoxazinone derivative 2.

Yellow crystals; Yield 50\%; m.p. $140-141{ }^{\circ} \mathrm{C}$; Anal. Calcd. for $\mathrm{C}_{21} \mathrm{H}_{15} \mathrm{NO}_{5} \mathrm{~S}$ : C, 64.11; H, 3.84; N, 3.56. Found: C, 64.12; $\mathrm{H}, 3.77$; N, 3.51; IR (KBr, cm ${ }^{-1}$ ): 1766 (CO of lactone), 1627 $(\mathrm{C}=\mathrm{N}), 1370\left(\mathrm{SO}_{3}\right) ;{ }^{1} \mathrm{H}-\mathrm{NMR}(500 \mathrm{MHz}, \mathrm{DMSO}-\mathrm{d} 6, \delta /$ ppm): $2.4\left(s, 3 \mathrm{H}, \mathrm{CH}_{3}\right), 8.21(d, 1 \mathrm{H}, \mathrm{Ar}-\mathrm{H}), 8.09(t, 1 \mathrm{H}$, $\operatorname{Ar}-\mathrm{H}), 7.79$ ( $t, 1 \mathrm{H}, \mathrm{Ar}-\mathrm{H}), 7.66(d, 1 \mathrm{H}, \mathrm{Ar}-\mathrm{H}), 7.96(d, 2 \mathrm{H}$, $\operatorname{Ar}-\mathrm{H}), 7.02$ (d, 2H, Ar-H), $7.74(d, 2 \mathrm{H}, \mathrm{Ar}-\mathrm{H}), 7.40(d, 2 \mathrm{H}$, Ar-H); ${ }^{13} \mathrm{C}-\mathrm{NMR}(400 \mathrm{MHz}$, DMSO-d6, $\delta / \mathrm{ppm}): 159(\mathrm{C}=\mathrm{O})$, 156.3 $(\mathrm{C}=\mathrm{N}), 21.3\left(\mathrm{CH}_{3}\right), 128.2,124.1,135.2,126.2,146.1$, $130.6,116.0,126.7,130.3,132.4,138.2,122.4,152.5$ (aromatic); MS (m/z, (relative abundance, \%)): $393\left(\mathrm{M}^{+}\right.$, 29.24), 374 (22.5), 261 (17), 177 (33.5), 105 (35.3), 91 (100). Synthesis of 4-(4-benzo[4,5]imidazo[1,2-c] quinazoline-6-yl)phenyl-4-methylbenzenesulfonate (3) and 4(3-(2-aminophenyl)-4-oxo-3,4-dihydroquinazolin-2-yl) phenyl-4-methylbenzenesulfonate (4)

A mixture of benzoxazinone 2 (1.18 g, 0.003 mole), $o$-phenylenediamine $(0.32 \mathrm{~g}, 0.003 \mathrm{~mole})$ and freshly fused sodium acetate $(0.2 \mathrm{~g})$ was fused at $180^{\circ} \mathrm{C}$ and $/$ or at $100^{\circ} \mathrm{C}$ for $3 \mathrm{~h}$. In each case, the reaction mixture was cooled, washed with dil. $\mathrm{HCl}$. The separated solid product was dried and recrystallized from a mixture of ether-ethanol and methanol to give $\mathbf{3}$ and $\mathbf{4}$, respectively.

\section{Compound 3}

Brown crystals; Yield 61\%; m.p. 291-293 C; Anal. Calcd. for $\mathrm{C}_{27} \mathrm{H}_{19} \mathrm{~N}_{3} \mathrm{O}_{3} \mathrm{~S}$ : C, 69.66; H, 4.11; N, 9.03. Found: C, 69.33; H, 4.00; N, 9.00; IR (KBr, cm $\left.{ }^{-1}\right): 1600(\mathrm{C}=\mathrm{N}), 1360$ $\left(\mathrm{SO}_{3}\right) ;{ }^{1} \mathrm{H}-\mathrm{NMR}(500 \mathrm{MHz}, \mathrm{DMSO}-\mathrm{d} 6, \delta / \mathrm{ppm}): 2.4(\mathrm{~s}, 3 \mathrm{H}$, $\left.\mathrm{CH}_{3}\right), 7.84(d, 1 \mathrm{H}, \mathrm{Ar}-\mathrm{H}), 7.58(t, 1 \mathrm{H}, \mathrm{Ar}-\mathrm{H}), 7.83(t, 1 \mathrm{H}$, Ar-H), $8.16(d, 1 \mathrm{H}, \mathrm{Ar}-\mathrm{H}), 7.91(d, 2 \mathrm{H}, \mathrm{Ar}-\mathrm{H}), 7.01(d, 2 \mathrm{H}$, $\operatorname{Ar}-\mathrm{H}), 7.59$ (d, 2H, Ar-H), $7.22(m, 2 \mathrm{H}, \mathrm{Ar}-\mathrm{H}), 7.56(d, 1 \mathrm{H}$, Ar-H), 7.74 (d, 2H, Ar-H), 7.40 (d, 2H, Ar-H); ${ }^{13} \mathrm{C}-\mathrm{NMR}(400 \mathrm{MHz}, \mathrm{DMSO}-\mathrm{d} 6, \delta / \mathrm{ppm}): 142.9,161(2 \mathrm{C}=\mathrm{N})$, 21.3( $\left(\mathrm{CH}_{3}\right), 122.8,127.7,132.2,128.5,149.3,118.7,144.0$, 120.1, 123.0, 112.1, 130.7, 116.4, 135.8, 150.2, 126.7, 130.3, 132.4, 138.2 (aromatic); MS (m/z, (relative abundance, $\%)$ ): $467\left(\mathrm{M}^{+}+2,0.96\right), 354$ (11.4), 255 (13.4), 167 (55.8), 105 (15.8), 91 (100).

\section{Compound 4}

Brown crystals; Yield 55\%; m.p. $170-172^{\circ} \mathrm{C}$, Anal. Calcd. for $\mathrm{C}_{27} \mathrm{H}_{21} \mathrm{~N}_{3} \mathrm{O}_{4} \mathrm{~S}$ : C, 67.07; H, 4.38; N, 8.69. Found: C, 67.00; H, 4.28; N, 8.34; IR ( KBr, cm $\left.{ }^{-1}\right): 3332-3425\left(\mathrm{NH}_{2}\right)$, 1665 (CO, amidic), $1600(\mathrm{C}=\mathrm{N}), 1360\left(\mathrm{SO}_{3}\right) ;{ }^{1} \mathrm{H}-\mathrm{NMR}(500$ $\mathrm{MHz}, \mathrm{DMSO}-\mathrm{d} 6, \delta$ / ppm): $2.4\left(\mathrm{~s}, 3 \mathrm{H}, \mathrm{CH}_{3}\right), 3.3\left(\mathrm{~s}, 2 \mathrm{H}, \mathrm{NH}_{2}\right)$, $8.03(d, 1 \mathrm{H}, \mathrm{Ar}-\mathrm{H}), 7.65(t, 1 \mathrm{H}, \mathrm{Ar}-\mathrm{H}), 7.70(t, 1 \mathrm{H}, \mathrm{Ar}-\mathrm{H})$, $7.63(d, 1 \mathrm{H}, \mathrm{Ar}-\mathrm{H}), 6.96(d, 1 \mathrm{H}, \mathrm{Ar}-\mathrm{H}), 7.40(t, 1 \mathrm{H}, \mathrm{Ar}-\mathrm{H})$, $7.56(d, 1 \mathrm{H}, \mathrm{Ar}-\mathrm{H}), 6.79(t, 1 \mathrm{H}, \mathrm{Ar}-\mathrm{H}), 7.40(d, 2 \mathrm{H}, \mathrm{Ar}-\mathrm{H})$, $7.02(d, 2 \mathrm{H}, \mathrm{Ar}-\mathrm{H}), 7.74$ (d, 2H, Ar-H), 7.45 (d, 2H, Ar-H); ${ }^{13} \mathrm{C}-\mathrm{NMR}(400 \mathrm{MHz}, \mathrm{DMSO}-\mathrm{d} 6, \delta / \mathrm{ppm}): 156(\mathrm{C}=\mathrm{N})$, 
21.3 $\left(\mathrm{CH}_{3}\right), 160(\mathrm{C}=\mathrm{O}), 126.6,127.3,133.4,126.7,148.7$, $118.7,120.8,126.8,134.1,114.5,125.1,118.9,114.8,131.8$, 116.0, 121.3, 151.6, 126.7, 130.3, 132.4, 138.2 (aromatic); MS (m/z, (relative abundance, \%)): $483\left(\mathrm{M}^{+}, 7.19\right), 389$ (31.5), 298 (3.3), 177 (45.3), 165 (3.3), 91 (100).

Synthesis of 4-[2-[(N-2-aminophenyl) benzoyl]carbamo yl]phenyl-4'-methylbenzenesulfonate (5)

A mixture of benzoxazinone $2(1.18 \mathrm{~g}, 0.003 \mathrm{~mole})$ and $o$-phenylenediamine $(0.32 \mathrm{gm}, 0.003 \mathrm{~mole})$ in ethanol $(20$ $\mathrm{mL}$ ) was refluxed for 8 hours. The solid product that separated on cooling was filtered off and recrystallized from ethanol to give $\mathbf{5}$.

White crystals; Yield $33 \%$; m.p. $225-227^{\circ} \mathrm{C}$; Anal. Calcd. for $\mathrm{C}_{27} \mathrm{H}_{23} \mathrm{~N}_{3} \mathrm{O}_{5} \mathrm{~S}$ : C, 64.66; H, 4.62; N, 8.38. Found: C, 64.42; H, 4.54; N, 8.45; IR (KBr, cm $\left.{ }^{-1}\right): 3332-3425\left(\mathrm{NH}_{2}\right)$, $3240(-\mathrm{NH}), 1665(\mathrm{CONH}), 1375\left(\mathrm{SO}_{3}\right) ;{ }^{1} \mathrm{H}-\mathrm{NMR}(500 \mathrm{MHz}$, DMSO-d6, $\delta$ / ppm): $2.4\left(s, 3 \mathrm{H}, \mathrm{CH}_{3}\right), 8.4\left(s, 2 \mathrm{H}, \mathrm{NH}_{2}\right), 8.6$ $(s, 2 \mathrm{H},-2 \mathrm{NHCO}), 7.87(d, 1 \mathrm{H}, \mathrm{Ar}-\mathrm{H}), 7.37(t, 1 \mathrm{H}, \mathrm{Ar}-\mathrm{H})$, $7.68(t, 1 \mathrm{H}, \mathrm{Ar}-\mathrm{H}), 8.37(d, 1 \mathrm{H}, \mathrm{Ar}-\mathrm{H}), 6.98(d, 1 \mathrm{H}, \mathrm{Ar}-\mathrm{H})$, $7.40(t, 1 \mathrm{H}, \mathrm{Ar}-\mathrm{H}), 6.79(t, 1 \mathrm{H}, \mathrm{Ar}-\mathrm{H}), 8(t, 1 \mathrm{H}, \mathrm{Ar}-\mathrm{H}), 7.86$ (d, 2H, Ar-H), $7.13(d, 2 \mathrm{H}, \mathrm{Ar}-\mathrm{H}), 7.74(d, 2 \mathrm{H}, \mathrm{Ar}-\mathrm{H}), 7.40$ (d, 2H, Ar-H); ${ }^{13} \mathrm{C}-\mathrm{NMR}(400 \mathrm{MHz}, \mathrm{DMSO}-\mathrm{d} 6, \delta / \mathrm{ppm})$ : $164.5,167.7(2 \mathrm{CONH}), 21.3\left(\mathrm{CH}_{3}\right), 127.6,124.4,132.4$, $119.4,138.9,123.2,122.8,149.5,114.5,125.1,118.9,125.5$, $128.8,116.0,126.8,153.6,126.6,130.3,132.4,138.1$ (aromatic);MS (m/z, (relative abundance, \%)): $501\left(\mathrm{M}^{+}\right.$, 4.13), 479 (1.8), 382 (22.5), 198 (12.4), 115 (100), 86 (14.9). Synthesis of 4-(4-oxo-3,4-dihydroquinazolin-2-yl)phenyl -4-methyl benzenesulfonate (6)

A mixture of benzoxazinone $2(3.93 \mathrm{~g}, 0.01 \mathrm{~mole})$ and ammonium acetate $(2.68 \mathrm{~g}, 0.01 \mathrm{~mole})$ was fused in an oil bath at $160-170^{\circ} \mathrm{C}$ for $3 \mathrm{~h}$. The reaction mixture was left to cool, washed with water several times, filtered off, dried and recrystallized from ethanol to give $\mathbf{6}$.

Grey crystals; Yield 55\%; m.p. $215-217^{\circ} \mathrm{C}$; Anal. Calcd. for $\mathrm{C}_{21} \mathrm{H}_{16} \mathrm{~N}_{2} \mathrm{O}_{4} \mathrm{~S}: \mathrm{C}, 64.27 ; \mathrm{H}, 4.11 ; \mathrm{N}, 7.14$. Found: C, 64.15; H, 4.06; N, 7.06; IR $\left(\mathrm{KBr}, \mathrm{cm}^{-1}\right): 3336(\mathrm{OH}), 3173$ $(-\mathrm{NH}), 1661(\mathrm{CONH}), 1604(\mathrm{CN}), 1377\left(\mathrm{SO}_{3}\right) ;{ }^{1} \mathrm{H}-\mathrm{NMR}$ (500 MHz, DMSO-d6, $\delta$ / ppm): $2.4\left(s, 3 \mathrm{H}, \mathrm{CH}_{3}\right), 3.5(s, 1 \mathrm{H}$, $\mathrm{NH}), 8.03(d, 1 \mathrm{H}, \mathrm{Ar}-\mathrm{H}), 7.65(t, 1 \mathrm{H}, \mathrm{Ar}-\mathrm{H}), 7.70(t, 1 \mathrm{H}$, $\operatorname{Ar}-\mathrm{H}), 7.63(d, 1 \mathrm{H}, \mathrm{Ar}-\mathrm{H}), 7.40(d, 2 \mathrm{H}, \mathrm{Ar}-\mathrm{H}), 7.02(d, 2 \mathrm{H}$, Ar-H $), \quad 7.74$ (d, 2H, Ar-H), 7.75 (d, 2H, Ar-H); ${ }^{13} \mathrm{C}-\mathrm{NMR}(400 \mathrm{MHz}, \mathrm{DMSO}-\mathrm{d} 6, \delta / \mathrm{ppm}): 161.0(\mathrm{C}=\mathrm{O})$, $152.3(\mathrm{C}=\mathrm{N}), 21.3\left(\mathrm{CH}_{3}\right), 126.6,127.4,133.4,126.7,148.9$, 120.8, 131.8, 116.0, 125.0, 151.6, 126.9, 130.4, 132.4, 138.2 (aromatic);MS (m/z, (relative abundance, \%)): $392\left(\mathrm{M}^{+}\right.$, 67.35), 356 (4.6), 287 (55.3), 178 (13.4), 105 (100), 85 (34.5).

Synthesis of 4-(3-(1,5-dimethyl-3-oxo-2-phenyl -2,3- dihyhro-1 $H$-pyrazol-4-yl)-4-oxo-3,4-dihydroquinazolin-2 -yl) phenyl-4-methyl benzene sulfonate (7)

A mixture of $2(0.79 \mathrm{~g}, 0.002$ mole) and 4-aminoantipyrin $(0.41 \mathrm{~g}, 0.002 \mathrm{~mole})$ in glacial acetic acid $(20 \mathrm{~mL})$ was refluxed for 6 hours then cooled. The reaction mixture was poured onto ice water; the precipitated product was filtered off and recrystallized from acetic acid to give product 7 .

White crystals; Yield 83\%; m.p. 195-197 C; Anal. Calcd. for $\mathrm{C}_{32} \mathrm{H}_{26} \mathrm{~N}_{4} \mathrm{O}_{5} \mathrm{~S}$ : C, 66.42; H, 4.53; N, 9.68. Found: C, 66.33; H, 4.48; N, 9.63; IR (KBr, cm $\left.{ }^{-1}\right)$ : 1665 (CO, amidic), $1613(\mathrm{CN}), 1380\left(\mathrm{SO}_{3}\right) ;{ }^{1} \mathrm{H}-\mathrm{NMR}(500 \mathrm{MHz}, \mathrm{DMSO}-\mathrm{d} 6, \delta /$ ppm): $2.4\left(s, 3 \mathrm{H}, \mathrm{CH}_{3}\right), 3.6\left(s, 3 \mathrm{H}, \mathrm{CH}_{3}\right), 2.7\left(s, 3 \mathrm{H}, \mathrm{CH}_{3}\right)$, $8.03(d, 1 \mathrm{H}, \mathrm{Ar}-\mathrm{H}), 7.65(t, 1 \mathrm{H}, \mathrm{Ar}-\mathrm{H}), 7.70(t, 1 \mathrm{H}, \mathrm{Ar}-\mathrm{H})$, $7.63(d, 1 \mathrm{H}, \mathrm{Ar}-\mathrm{H}), 7.35(d, 2 \mathrm{H}, \mathrm{Ar}-\mathrm{H}), 7.37(t, 2 \mathrm{H}, \mathrm{Ar}-\mathrm{H})$, $6.09(t, 1 \mathrm{H}, \mathrm{Ar}-\mathrm{H}), 7.40(d, 2 \mathrm{H}, \mathrm{Ar}-\mathrm{H}), 7.02(d, 2 \mathrm{H}, \mathrm{Ar}-\mathrm{H})$, $7.74(d, 2 \mathrm{H}, \mathrm{Ar}-\mathrm{H}), 7.42(d, 2 \mathrm{H}, \mathrm{Ar}-\mathrm{H}) ;{ }^{13} \mathrm{C}-\mathrm{NMR}(400 \mathrm{MHz}$, DMSO-d6, $\delta / \mathrm{ppm}): 160.7,165.2(2 \mathrm{C}=\mathrm{O}), 155.9(\mathrm{C}=\mathrm{N}), 21.3$, $25.6\left(2 \mathrm{CH}_{3}\right), 103.4,133.7(\mathrm{C}=\mathrm{C}), 35.6\left(\mathrm{~N}^{-} \mathrm{CH}_{3}\right), 126.6,127.4$, 133.4, 126.7, 148.9, 120.8, 131.8, 116.0, 125.0, 151.6, 126.9, $130.4,132.4,138.2,123.2,129.2,133.9,122.8$ (aromatic); MS (m/z, (relative abundance, \%)): $578\left(\mathrm{M}^{+}, 4.00\right), 445$ (16.5), 397 (44.2), 258 (35.3), 175 (100), 117 (25.7).

Synthesis of 4-(4-oxo-3-(pyridin-2-yl)-3,4 dihydroquinazolin-2-yl)phenyl 4-methyl benzenesulfonate (8)

A mixture of benzoxazinone 2 (3.93 g, 0.01 mole) and 2-aminopyridine ( $0.94 \mathrm{~g}, 0.01 \mathrm{~mole})$ was fused in an oil bath at $150-155^{\circ} \mathrm{C}$ in presence of anhydrous $\mathrm{ZnCl}_{2}(1 \mathrm{~g})$ for $4 \mathrm{~h}$. The reaction mixture was triturated with ice $/ \mathrm{HCl}$. The formed solid product was filtered off, washed with water several times, dried and recrystallized from methanol to give 8.

White crystals; Yield $68 \%$; m.p. $88-90^{\circ} \mathrm{C}$; Anal. Calcd. for $\mathrm{C}_{26} \mathrm{H}_{19} \mathrm{~N}_{3} \mathrm{O}_{4} \mathrm{~S}: \mathrm{C}, 66.51 ; \mathrm{H}, 4.08 ; \mathrm{N}, 8$.95. Found: C, 66.34; H, 4.06; N, 8.68; IR $\left(\mathrm{KBr}, \mathrm{cm}^{-1}\right): 1685(\mathrm{CO}$, amidic), $1597(\mathrm{CN})$, $1374\left(\mathrm{SO}_{3}\right) ;{ }^{1} \mathrm{H}-\mathrm{NMR}(500 \mathrm{MHz}, \mathrm{DMSO}-\mathrm{d} 6, \delta / \mathrm{ppm}): 2.4(s$, $\left.3 \mathrm{H}, \mathrm{CH}_{3}\right), 8.03(d, 1 \mathrm{H}, \mathrm{Ar}-\mathrm{H}), 7.65(t, 1 \mathrm{H}, \mathrm{Ar}-\mathrm{H}), 7.70(t, 1 \mathrm{H}$, $\operatorname{Ar}-\mathrm{H}), 7.63(d, 1 \mathrm{H}, \operatorname{Ar}-\mathrm{H}), 6.70(d, 1 \mathrm{H}, \operatorname{Ar}-\mathrm{H}), 7.55(t, 1 \mathrm{H}$, Ar-H), $6.62(t, 1 \mathrm{H}, \mathrm{Ar}-\mathrm{H}), 8.07(d, 1 \mathrm{H}, \mathrm{Ar}-\mathrm{H}) 7.40(d, 2 \mathrm{H}$, $\operatorname{Ar}-\mathrm{H}), 7.02(d, 2 \mathrm{H}, \mathrm{Ar}-\mathrm{H}), 7.74(d, 2 \mathrm{H}, \mathrm{Ar}-\mathrm{H}), 7.42(d, 2 \mathrm{H}$, $\mathrm{Ar}-\mathrm{H}) ;{ }^{13} \mathrm{C}-\mathrm{NMR}(400 \mathrm{MHz}, \quad$ DMSO-d6, $\delta \quad / \mathrm{ppm})$ : $160.7(\mathrm{C}=\mathrm{O}), 156.2(\mathrm{C}=\mathrm{N}), 21.3\left(\mathrm{CH}_{3}\right), 126.6,127.4,133.4$, $126.7,148.9,120.8,131.8,116.0,125.0,151.6,126.9,130.4$, 132.4, 138.2, 123.9, 147.6, 138.3, 117.9, 148.1 (aromatic); MS (m/z, (relative abundance, \%)): $469\left(\mathrm{M}^{+}, 3.24\right), 433$ (6.7), 356 (55.7), 248 (25.5), 105 (100), 86 (33.8).

Synthesis of 4-(4-oxo-3-(pyridine-3-yl)-3,4-dihydroquinazolin-2-yl) phenyl 4-methyl benzenesulfonate (9)

A mixture of benzoxazinone $2(3.93 \mathrm{~g}, 0.01 \mathrm{~mole})$ and 3 -aminopyridine ( $0.94 \mathrm{~g}, 0.01 \mathrm{~mole})$ was fused in an oil bath at $150-155^{\circ} \mathrm{C}$ in presence of anhydrous $\mathrm{ZnCl}_{2}(1 \mathrm{~g})$ for $4 \mathrm{~h}$. The reaction mixture was triturated with ice $/ \mathrm{HCl}$. The formed solid product was filtered off, washed with water several times, dried and recrystallized from methanol to give 9.

White crystals; Yield 79\%; m.p. $112-114^{\circ} \mathrm{C}$; Anal. Calcd. for $\mathrm{C}_{26} \mathrm{H}_{19} \mathrm{~N}_{3} \mathrm{O}_{4} \mathrm{~S}$ : C, 66.51; H, 4.08; N, 8.95. Found: C, 66.22; H, 3.96; N, 8.87; IR (KBr, cm $\left.{ }^{-1}\right): 1685$ (CO, amidic), $1597(\mathrm{CN}), 1374\left(\mathrm{SO}_{3}\right) ;{ }^{1} \mathrm{H}-\mathrm{NMR}(500 \mathrm{MHz}$, DMSO-d6, $\delta$ / ppm): $2.4\left(s, 3 \mathrm{H}, \mathrm{CH}_{3}\right), 8.03(d, 1 \mathrm{H}, \mathrm{Ar}-\mathrm{H}), 7.63(t, 1 \mathrm{H}$, Ar-H), $7.70(t, 1 \mathrm{H}, \operatorname{Ar}-\mathrm{H}), 7.63(d, 1 \mathrm{H}, \mathrm{Ar}-\mathrm{H}), 7.27(d, 1 \mathrm{H}$, Ar-H), $7.36(t, 1 \mathrm{H}, \mathrm{Ar}-\mathrm{H}), 8.09(d, 1 \mathrm{H}, \mathrm{Ar}-\mathrm{H}), 8.02(s, 1 \mathrm{H}$, $\operatorname{Ar}-\mathrm{H}), 7.40(d, 2 \mathrm{H}, \operatorname{Ar}-\mathrm{H}), 7.02(d, 2 \mathrm{H}, \operatorname{Ar}-\mathrm{H}), 7.74(d, 2 \mathrm{H}$, Ar-H), $7.42(d, 2 \mathrm{H}, \mathrm{Ar}-\mathrm{H}) ;{ }^{13} \mathrm{C}-\mathrm{NMR}(400 \mathrm{MHz}, \mathrm{DMSO}-\mathrm{d} 6$, $\delta / \mathrm{ppm}): 160.7(\mathrm{C}=\mathrm{O}), 156.2,138.8(2 \mathrm{C}=\mathrm{N}), 21.3\left(\mathrm{CH}_{3}\right)$, 126.6, 127.4, 133.4, 126.7, 148.9, 120.8, 131.8, 116.0, 125.0, 
151.6, 126.9, 130.4, 132.4, 138.2, 122.8, 145.1, 137.5, 124.7 (aromatic); MS (m/z, (relative abundance, \%)): $469\left(\mathrm{M}^{+}\right.$, 5.88), 446 (13.3), 366 (37.6), 237 (38.5), 165 (100), 112 (43.7).

Synthesis of 4-(4-thioxo-4H-3,1-benzothiazin-2-yl)phenyl -4-methyl benzenesulfonate(10)

A mixture of benzoxazinone 2 (3.93g, 0.01 mole) and phosphorous pentasulphide $(8.9 \mathrm{~g}, 0.02$ mole) in dry xylene $(40 \mathrm{~mL})$ was refluxed for $8 \mathrm{~h}$. The reaction mixture was filtered off while hot, concentrated and the solid that separated on cooling was washed with petroleum ether (b.p. $80-100^{\circ}$ ), then recrystallized from ethanol to give $\mathbf{1 0}$.

Yellow crystals; Yield 38\%; m.p. $135-137^{\circ} \mathrm{C}$; Anal. Calcd. for $\mathrm{C}_{21} \mathrm{H}_{15} \mathrm{NO}_{3} \mathrm{~S}_{3}$ : C, 59.27; $\mathrm{H}, 3.55 ; \mathrm{N}, 3.29$. Found: $\mathrm{C}$, 59.18; H, 3.50; N, 3.24; IR (KBr, cm $\left.{ }^{-1}\right): 1323$ (CS), 1595 $(\mathrm{C}=\mathrm{N}), 1364\left(\mathrm{SO}_{3}\right) .{ }^{1} \mathrm{H}-\mathrm{NMR}(500 \mathrm{MHz}, \mathrm{DMSO}-\mathrm{d} 6, \delta /$ ppm): $2.4\left(s, 3 \mathrm{H}, \mathrm{CH}_{3}\right), 7.34(d, 1 \mathrm{H}, \mathrm{Ar}-\mathrm{H}), 7.45(m, 3 \mathrm{H}$, $\mathrm{Ar}-\mathrm{H}), 7.66$ (d, 2H, Ar-H), $7.02(d, 2 \mathrm{H}, \mathrm{Ar}-\mathrm{H}), 7.74(d, 2 \mathrm{H}$, Ar-H), 7.42 (d, 2H, Ar-H); ${ }^{13} \mathrm{C}-\mathrm{NMR}(400 \mathrm{MHz}, \mathrm{DMSO}-\mathrm{d} 6$, $\delta / \mathrm{ppm}): 218(\mathrm{C}=\mathrm{S}), 165.0(\mathrm{C}=\mathrm{N}), 21.3\left(\mathrm{CH}_{3}\right), 131.0,126.6$, 132.0, 126.0, 153.3, 135.4, 130.6, 116.0, 128.8, 152.5, 126.7, $130.3,132.4,138.2$ (aromatic); MS (m/z, (relative abundance, \%)): $425\left(\mathrm{M}^{+}, 24.46\right), 385$ (24.5), 256 (7.8), 227 (42.6), 187 (100), 91 (33.5).

Synthesis of 4-(3-hydroxy-4-oxo-3,4-dihydroquinazo-lin2-yl)phenyl -4-methylbenzenesulfonate (11)

To a solution of benzoxazinone $2(2.35 \mathrm{~g}, 0.006 \mathrm{~mole})$ in ethanol $(30 \mathrm{~mL})$, hydroxylamine hydrochloride $(0.417 \mathrm{~g}$, 0.006 mole $)$ and sodium acetate $(0.49 \mathrm{~g}, 0.006$ mole $)$ dissolved in the least amount of water was added. The reaction mixture was refluxed for $8 \mathrm{~h}$, cooled and then concentrated. The solid product that separated on cooling was filtered off and recrystallized from ethanol to give $\mathbf{1 1}$.

Yellow crystals; Yield 62\%; m.p. $170-172^{\circ} \mathrm{C}$; Anal. Calcd. for $\mathrm{C}_{21} \mathrm{H}_{16} \mathrm{~N}_{2} \mathrm{O}_{5} \mathrm{~S}$ : C, 61.76; H, 3.95; N, 6.86. Found: $\mathrm{C}$, 61.66; H, 4.00; N, 6.84; IR (KBr, cm $\left.{ }^{-1}\right): 3220(\mathrm{OH}), 1665$ $(\mathrm{CON}), 1613(\mathrm{C}=\mathrm{N}), 1380\left(\mathrm{SO}_{3}\right) ;{ }^{1} \mathrm{H}-\mathrm{NMR}(500 \mathrm{MHz}$, DMSO-d6, $\delta / \mathrm{ppm}): 2.4\left(s, 3 \mathrm{H}, \mathrm{CH}_{3}\right), 3.7(s, 1 \mathrm{H}, \mathrm{OH}), 8.03$ $(d, 1 \mathrm{H}, \mathrm{Ar}-\mathrm{H}), 7.65(t, 1 \mathrm{H}, \mathrm{Ar}-\mathrm{H}), 7.70(t, 1 \mathrm{H}, \mathrm{Ar}-\mathrm{H}), 7.63(d$, $1 \mathrm{H}, \mathrm{Ar}-\mathrm{H}), 7.40(d, 2 \mathrm{H}, \mathrm{Ar}-\mathrm{H}), 7.02(d, 2 \mathrm{H}, \mathrm{Ar}-\mathrm{H}), 7.74(d$, $2 \mathrm{H}, \mathrm{Ar}-\mathrm{H}), 7.42(d, 2 \mathrm{H}, \mathrm{Ar}-\mathrm{H}) ;{ }^{13} \mathrm{C}-\mathrm{NMR}(400 \mathrm{MHz}$, DMSO-d6, $\delta / \mathrm{ppm}): 155(\mathrm{C}=\mathrm{O}), 156.2(\mathrm{C}=\mathrm{N}), 21.3\left(\mathrm{CH}_{3}\right)$, 126.6, 127.3, 133.4, 126.7, 148.7, 120.8, 131.8, 116.5, 121.2, 151.6, 126.7, 130.3, 132.4, 138.2 (aromatic); MS (m/z, (relative abundance, \%)): $408\left(\mathrm{M}^{+}, 5.70\right), 356$ (4.15), 243 (23.4), 167 (53.2), 105 (100), 86 (24.7).

Synthesis of 4-(3-amino-4-oxo-3,4-dihydroquinazo lin-2yl)phenyl -4 -methylbenzenesulfonate (12)

A solution of benzoxazinone 2 (3.93 g, 0.01 mole) and hydrazine hydrate $(1.0 \mathrm{~g}, 0.02$ mole) in absolute ethanol (30 $\mathrm{mL}$ ) was refluxed for 6 hours. The solid product that separated on cooling was filtered off, dried and recrystallized from ethanol to afford the quinazolinone derivative $\mathbf{1 2}$.

Yellow crystals; Yield $60 \%$; m.p. $156-158^{\circ} \mathrm{C}$; Anal. Calcd. for $\mathrm{C}_{21} \mathrm{H}_{17} \mathrm{~N}_{3} \mathrm{O}_{4} \mathrm{~S}$ : C, 61.90; H, 4.21; N, 10.31. Found: $\mathrm{C}$, 61.80; H, 4.20; N, 10.22; IR $\left(\mathrm{KBr}, \mathrm{cm}^{-1}\right): 3332-3425\left(\mathrm{NH}_{2}\right)$, $1653(\mathrm{CON}), 1597(\mathrm{C}=\mathrm{N}), 1360\left(\mathrm{SO}_{3}\right) ;{ }^{1} \mathrm{H}-\mathrm{NMR}(500 \mathrm{MHz}$,
DMSO-d6, $\delta$ / ppm): $2.4\left(s, 3 \mathrm{H}, \mathrm{CH}_{3}\right), 4.9\left(s, 2 \mathrm{H}, \mathrm{NH}_{2}\right), 8.03$ $(d, 1 \mathrm{H}, \mathrm{Ar}-\mathrm{H}), 7.65(t, 1 \mathrm{H}, \mathrm{Ar}-\mathrm{H}), 7.70(t, 1 \mathrm{H}, \mathrm{Ar}-\mathrm{H}), 7.63(d$, 1H, Ar-H), 7.40 (d, 2H, Ar-H), 7.02 (d, 2H, Ar-H), $7.74(d$, 2H, Ar-H), 7.42 (d, 2H, Ar-H); ${ }^{13} \mathrm{C}-\mathrm{NMR}(400 \mathrm{MHz}$, DMSO-d6, $\delta / \mathrm{ppm}): 155(\mathrm{C}=\mathrm{O}), 156.2(\mathrm{C}=\mathrm{N}), 21.3\left(\mathrm{CH}_{3}\right)$, 126.6, 127.3, 133.4, 126.7, 148.7, 120.8, 131.8, 116.5, 121.2, 151.6, 126.7, 130.3, 132.4, 138.2 (aromatic); MS (m/z, (relative abundance, \%)): $407\left(\mathrm{M}^{+}, 100\right), 386$ (15.65), 295 (3.8), 158 (11.2), 115 (10.1), 85 (34.3).

Synthesis of 4-(3-benzylideneami-no)-4-oxo-3,4- dihydroquinazolin-2-yl) phenyl-4-methyl benzenesulfonate (13)

To a solution of 12 ( $4.07 \mathrm{~g}, 0.01$ mole) in absolute ethanol (30 mL) containing few drops of piperidine, benzaldehyde (1.06 g, 0.01 mole) was added. The reaction mixture was refluxed for $5 \mathrm{~h}$, concentrated and left to cool. The precipitated product was filtered off and recrystallized from ethanol to give $\mathbf{1 3 .}$

Yellow crystals; Yield 62\%; m.p. $180-182^{\circ} \mathrm{C}$; Anal. Calcd. for $\mathrm{C}_{28} \mathrm{H}_{21} \mathrm{~N}_{3} \mathrm{O}_{4} \mathrm{~S}: \mathrm{C}, 67.88 ; \mathrm{H}, 4.24 ; \mathrm{N}, 14.85$. Found: $\mathrm{C}$, 67.56; H, 4.18; N, 14.82; IR (KBr, cm $\left.{ }^{-1}\right): 1678$ (CON), 1596 $(\mathrm{C}=\mathrm{N}), 1360\left(\mathrm{SO}_{3}\right) ;{ }^{1} \mathrm{H}-\mathrm{NMR}(500 \mathrm{MHz}, \mathrm{DMSO}-\mathrm{d} 6, \delta /$ ppm): $2.4\left(s, 3 \mathrm{H}, \mathrm{CH}_{3}\right), 9.0(s, 1 \mathrm{H},-\mathrm{N}=\mathrm{CH}), 8.03(d, 1 \mathrm{H}$, $\operatorname{Ar}-\mathrm{H}), 7.65(t, 1 \mathrm{H}, \mathrm{Ar}-\mathrm{H}), 7.70(t, 1 \mathrm{H}, \mathrm{Ar}-\mathrm{H}), 7.63(d, 1 \mathrm{H}$, $\operatorname{Ar}-\mathrm{H}), 7.83$ (d, 2H, Ar-H), 7.52 (m, 3H, Ar-H), $7.40(d, 2 \mathrm{H}$, $\operatorname{Ar}-\mathrm{H}), 7.02$ (d, 2H, Ar-H), 7.74 (d, 2H, Ar-H), 7.42 (d, 2H, $\mathrm{Ar}-\mathrm{H}) ;{ }^{13} \mathrm{C}-\mathrm{NMR}(400 \mathrm{MHz}, \quad \mathrm{DMSO}-\mathrm{d} 6, \delta / \mathrm{ppm})$ : 166.7 $(\mathrm{C}=\mathrm{O}), 153.6(\mathrm{C}=\mathrm{N}), 166.7(\mathrm{~N}=\mathrm{CH}), 21.3\left(\mathrm{CH}_{3}\right), 126.6$, 127.3, 133.4, 126.7, 148.7, 120.8, 131.8, 116.5, 121.2, 151.6, $126.7,130.3,132.4,138.2,133.7,129.2,128.8,131.0$ (aromatic); MS (m/z, (relative abundance, \%)): $495\left(\mathrm{M}^{+}\right.$, 0.68), 397 (26.85), 284 (24.6), 176 (51.2), 115 (10.1), 85 (11.31).

Synthesis of 4-(3-(3-chloro-2-oxo-4-phenylazetidin-1yl)-4-oxo-3,4-dihydroquinazolin-2-yl)phenyl-4-methy l benzene sulfonate (14)

A mixture of 13 (4.95 g, 0.01 mole), chloroacetyl chloride ( $1.13 \mathrm{~g}, 0.01 \mathrm{~mole})$ and triethyl amine (5 drops) in dry dioxane $(30 \mathrm{~mL})$ was heated under reflux for 8 hours. The solid product that separated on cooling was filtered off, dried and recrystallized from ethanol to give $\mathbf{1 4}$.

White crystals; Yield $42 \%$; m.p. $115-117^{\circ} \mathrm{C}$; Anal. Calcd. for $\mathrm{C}_{30} \mathrm{H}_{22} \mathrm{CIN}_{3} \mathrm{O}_{5} \mathrm{~S}$ : C, 62.99; H, 3.85; N, 17.14. Found: $\mathrm{C}$, 62.81; H, 3.64; N, 17.12; IR (KBr, cm $\left.{ }^{-1}\right): 1678$ (CON), 1596 $(\mathrm{C}=\mathrm{N}), 1360\left(\mathrm{SO}_{3}\right) ;{ }^{1} \mathrm{H}-\mathrm{NMR}(500 \mathrm{MHz}, \mathrm{DMSO}-\mathrm{d} 6, \delta /$ ppm): $2.4\left(s, 3 \mathrm{H}, \mathrm{CH}_{3}\right), 4.1(s, 1 \mathrm{H}, \mathrm{CH}), 3.5(s, 1 \mathrm{H}, \mathrm{CH}), 8.03$ $(d, 1 \mathrm{H}, \mathrm{Ar}-\mathrm{H}), 7.65(t, 1 \mathrm{H}, \mathrm{Ar}-\mathrm{H}), 7.70(t, 1 \mathrm{H}, \mathrm{Ar}-\mathrm{H}), 7.63(d$, $1 \mathrm{H}, \mathrm{Ar}-\mathrm{H}), 7.29(d, 2 \mathrm{H}, \mathrm{Ar}-\mathrm{H}), 7.45(t, 2 \mathrm{H}, \mathrm{Ar}-\mathrm{H}), 7.27(t$, 1H, Ar-H), 7.40 (d, 2H, Ar-H), 7.02 (d, 2H, Ar-H), $7.74(d$, $2 \mathrm{H}, \mathrm{Ar}-\mathrm{H}), 7.42(d, 2 \mathrm{H}, \mathrm{Ar}-\mathrm{H}) ;{ }^{13} \mathrm{C}-\mathrm{NMR}(400 \mathrm{MHz}$, DMSO-d6, $\delta / \mathrm{ppm}): 160.7,163.6(2 \mathrm{C}=\mathrm{O}), 156.2(\mathrm{C}=\mathrm{N})$, 70.6(N-CH), 76.6(CH-Cl), 21.3( $\left.\mathrm{CH}_{3}\right), 126.6,127.3,133.4$, 126.7, 148.7, 120.8, 131.8, 116.5, 121.2, 151.6, 126.7, 130.3, 132.4, 138.2 , 143.5, 126.9, 128.5, 126.7 (aromatic); MS (m/z, (relative abundance, \%)): $571\left(\mathrm{M}^{+}, 12.55\right), 453$ (30.45), 365 (100), 247 (33.1), 175 (17.1), 112 (23.1).

Synthesis of 4-(4- oxo-3-(4- oxo-2-phenyl thiazolidin 


\section{-3-yl)-3,4-dihydroquinazolin-2-yl)pheny-4-methyl benzenesulfonate (15)}

Thioglycolic acid ( $1.50 \mathrm{~g}, 0.01 \mathrm{~mole})$ in dry benzene (20 $\mathrm{mL}$ ) was added drop wise with stirring at room temperature to $13(4.95 \mathrm{~g}, 0.01 \mathrm{~mole})$ in dry benzene $(20 \mathrm{~mL})$ for 1 hour. The reaction mixture was heated under reflux for 6 hours, cooled and the precipitated product was filtered off and recrystallized from ethanol to give the desired product 15.

Yellow crystals; Yield $47 \%$; m.p. $80-85^{\circ} \mathrm{C}$; Anal. Calcd. for $\mathrm{C}_{30} \mathrm{H}_{23} \mathrm{~N}_{3} \mathrm{O}_{5} \mathrm{~S}_{2}$ : C, 63.27; H, 4.04; N, 17.07. Found: C, 63.15; H, 4.00; N, 17.02; IR (KBr, cm $\left.{ }^{-1}\right): 1678(\mathrm{CON}), 1596$ $(\mathrm{C}=\mathrm{N}), 1360\left(\mathrm{SO}_{3}\right) ;{ }^{1} \mathrm{H}-\mathrm{NMR}(500 \mathrm{MHz}$, DMSO-d6, $\delta /$ ppm): $2.4\left(s, 3 \mathrm{H}, \mathrm{CH}_{3}\right), 4.9(s, 1 \mathrm{H}, \mathrm{CH}), 3.9\left(s, 2 \mathrm{H}, \mathrm{CH}_{2}\right)$, $8.03(d, 1 \mathrm{H}, \mathrm{Ar}-\mathrm{H}), 7.65(t, 1 \mathrm{H}, \mathrm{Ar}-\mathrm{H}), 7.70(t, 1 \mathrm{H}, \mathrm{Ar}-\mathrm{H})$, $7.63(d, 1 \mathrm{H}, \mathrm{Ar}-\mathrm{H}), 7.36(d, 2 \mathrm{H}, \mathrm{Ar}-\mathrm{H}), 7.33(t, 2 \mathrm{H}, \mathrm{Ar}-\mathrm{H})$, $7.27(t, 1 \mathrm{H}, \mathrm{Ar}-\mathrm{H}), 7.40(d, 2 \mathrm{H}, \mathrm{Ar}-\mathrm{H}), 7.02(d, 2 \mathrm{H}, \mathrm{Ar}-\mathrm{H})$, $7.74(d, 2 \mathrm{H}, \mathrm{Ar}-\mathrm{H}), 7.42(d, 2 \mathrm{H}, \mathrm{Ar}-\mathrm{H}) ;{ }^{13} \mathrm{C}-\mathrm{NMR}(400 \mathrm{MHz}$, DMSO-d6, $\delta / \mathrm{ppm}): 160.7,163.6(2 \mathrm{C}=\mathrm{O}), 156.2(\mathrm{C}=\mathrm{N})$, 35.6( $\left(\mathrm{CH}_{2}-\mathrm{S}\right), 62.2(\mathrm{CH}-\mathrm{S}), 21.3\left(\mathrm{CH}_{3}\right), 126.6,127.3,133.4$, $126.7,148.7,120.8,131.8,116.5,121.2,151.6,126.7,130.3$, 132.4, 138.2, 128.6, 126.9, 127.1, 139.2 (aromatic); MS (m/z, (relative abundance, \%)): $569\left(\mathrm{M}^{+}, 10.34\right), 481$ (5.45), 397 (23.18), 288 (51.2), 185 (100), 91 (24.2).

Synthesis of 4-(3,3-diacetamido-4-oxo-3,4-dihydro quinazolin-2-yl) phenyl-4- methylbenzenesulfonate (16) and 4-(4-0xo-3,3-(dibenzamido)-3,4-dihydroquinazolin -2-yl)phenyl- 4- methyl benzenesulfonate (17)

A solution of quinazolinone 12 (4.07 g, 0.01 mole), acetyl chloride (1.56 g, 0.02 mole) and/or benzoyl chloride ( $2.8 \mathrm{~g}$, 0.02 mole) in dry pyridine $(30 \mathrm{~mL})$ was heated under reflux for 3 hours. The reaction mixture was cooled, then poured over ice/ $\mathrm{HCl}$ and the solid that separated out was filtered off, washed with water several times, dried and then recrystallized from methanol to afford $\mathbf{1 6}$ and $\mathbf{1 7}$, respectively.

\section{Compound 16}

White crystals; Yield $82 \%$; m.p. $145-147^{\circ} \mathrm{C}$; Anal. Calcd. for $\mathrm{C}_{25} \mathrm{H}_{21} \mathrm{~N}_{3} \mathrm{O}_{6} \mathrm{~S}: \mathrm{C}, 61.10 ; \mathrm{H}, 4.28 ; \mathrm{N}, 14.73$. Found: $\mathrm{C}$, 61.24; H, 4.16; N, 14.72; IR $\left(\mathrm{KBr}, \mathrm{cm}^{-1}\right): 1701-1736$ $(\mathrm{N}-\mathrm{C}=\mathrm{O}), 1653(\mathrm{CON}), 1597(\mathrm{C}=\mathrm{N}), 1360\left(\mathrm{SO}_{3}\right) ;{ }^{1} \mathrm{H}-\mathrm{NMR}$ (500 MHz, DMSO-d6, $\delta / \mathrm{ppm}): 2.4\left(s, 3 \mathrm{H}, \mathrm{CH}_{3}\right), 2.27(s, 6 \mathrm{H}$, $\left.-\mathrm{COCH}_{3}\right), 8.03(d, 1 \mathrm{H}, \mathrm{Ar}-\mathrm{H}), 7.65(t, 1 \mathrm{H}, \mathrm{Ar}-\mathrm{H}), 7.70(t, 1 \mathrm{H}$, Ar-H), $7.63(d, 1 \mathrm{H}, \operatorname{Ar}-\mathrm{H}), 7.40(d, 2 \mathrm{H}, \mathrm{Ar}-\mathrm{H}), 7.02(d, 2 \mathrm{H}$, $\mathrm{Ar}-\mathrm{H}), 7.74(d, 2 \mathrm{H}, \mathrm{Ar}-\mathrm{H}), 7.42(d, 2 \mathrm{H}, \mathrm{Ar}-\mathrm{H}) ;{ }^{13} \mathrm{C}-\mathrm{NMR}(4$ $00 \mathrm{MHz}$, DMSO-d6, $\delta / \mathrm{ppm}): 160.7,156.6(3 \mathrm{C}=\mathrm{O})$, 156.2(C $=\mathrm{N}), 21.3,20.8\left(3 \mathrm{CH}_{3}\right), 126.6,127.3,133.4,126.7$, 148.7, 120.8, 131.8, 116.5, 121.2, 151.6, 126.7, 130.3, 132.4, 138.2 (aromatic); MS (m/z, (relative abundance, \%)): 491 $\left(\mathrm{M}^{+}, 5.47\right), 386$ (23.45), 285 (11.8), 168 (100), 122 (13.5), 91 (12.4).

\section{Compound 17}

White crystals; Yield $84 \%$; m.p. $160-162^{\circ} \mathrm{C}$; Anal. Calcd. for $\mathrm{C}_{35} \mathrm{H}_{25} \mathrm{~N}_{3} \mathrm{O}_{6} \mathrm{~S}: \mathrm{C}, 68.29 ; \mathrm{H}, 4.07 ; \mathrm{N}, 18.45$. Found: $\mathrm{C}$, $68.09 ; \mathrm{H}, 4.00 ; \mathrm{N}, 18.42 ; \mathrm{IR}\left(\mathrm{KBr}, \mathrm{cm}^{-1}\right): 1701-1736$ $(\mathrm{N}-\mathrm{C}=\mathrm{O}), 1653(\mathrm{CON}), 1597(\mathrm{C}=\mathrm{N}), 1360\left(\mathrm{SO}_{3}\right) ;{ }^{13} \mathrm{C}-\mathrm{NM}$ $\mathrm{R}(400 \mathrm{MHz}$, DMSO-d6, $\delta / \mathrm{ppm}): 160.7,172.0(3 \mathrm{C}=\mathrm{O})$, 156.2(C $=\mathrm{N}), 21.3\left(\mathrm{CH}_{3}\right), 126.6,127.3,133.4,126.7,148.7$, $120.8,131.8,116.5,121.2,151.6,126.7,130.3,132.4,138.2$,
127.5, 128.8, 132.1, 134.3 (aromatic); MS (m/z, (relative abundance, \%)): $615\left(\mathrm{M}^{+}, 5.47\right), 566$ (3.65), 376 (100), 288 (11.45), 182 (3.8), $113(56.4)$.

Synthesis of 4-(3-(1,3-dioxoisoindolin-2-yl)-4-0xo -3,4dihydroquin-azolin-2-yl) phenyl-4-methylbenzene sulfonate (18)

A mixture of quinazolinone 12 (4.07 g, 0.01 mole) and phthalic anhydride (1.48 g, 0.01 mole) was fused in an oil bath at $150-160^{\circ} \mathrm{C}$ for 6 hours. The reaction mixture was triturated with ice/ $\mathrm{HCl}$. The solid product was filtered off, washed with water several times, dried and then recrystallized from ethanol affording $\mathbf{1 8 .}$

Brown crystals; Yield $65 \%$; m.p. $175-177^{\circ} \mathrm{C}$; Anal. Calcd. for $\mathrm{C}_{29} \mathrm{H}_{19} \mathrm{~N}_{3} \mathrm{O}_{6} \mathrm{~S}$ : C, 64.80; H, 3.54; N, 16.11. Found: $\mathrm{C}$, 64.66; H, 3.39; N, 16.12; IR (KBr, $\left.\mathrm{cm}^{-1}\right): 1744-1797(2 \mathrm{C}=\mathrm{O})$, $1704(\mathrm{CON}), 1601(\mathrm{C}=\mathrm{N}), 1376\left(\mathrm{SO}_{3}\right) ;{ }^{1} \mathrm{H}-\mathrm{NMR}(500 \mathrm{MHz}$, DMSO-d6, $\delta$ / ppm): $2.4\left(s, 3 \mathrm{H}, \mathrm{CH}_{3}\right), 8.03(d, 1 \mathrm{H}, \mathrm{Ar}-\mathrm{H})$, $7.65(t, 1 \mathrm{H}, \mathrm{Ar}-\mathrm{H}), 7.70(t, 1 \mathrm{H}, \mathrm{Ar}-\mathrm{H}), 7.63(d, 1 \mathrm{H}, \mathrm{Ar}-\mathrm{H})$, 7.85-7.88 (m, 4H, Ar-H), $7.40(d, 2 \mathrm{H}, \operatorname{Ar}-\mathrm{H}), 7.02(d, 2 \mathrm{H}$, $\mathrm{Ar}-\mathrm{H}), 7.74(d, 2 \mathrm{H}, \mathrm{Ar}-\mathrm{H}), 7.42(d, 2 \mathrm{H}, \mathrm{Ar}-\mathrm{H}) ;{ }^{13} \mathrm{C}-\mathrm{NMR}(4$ $00 \mathrm{MHz}$, DMSO-d6, $\delta / \mathrm{ppm}): 160.7,164.0(3 \mathrm{C}=\mathrm{O})$, 156.2(C $=\mathrm{N}), 21.3\left(\mathrm{CH}_{3}\right), 126.6,127.3,133.4,126.7,148.7$, $120.8,131.8,116.5,121.2,151.6,126.7,130.3,132.4,138.2$, 132.0, 123.7, 132.2 (aromatic); MS (m/z, (relative abundance, \%)): $537\left(\mathrm{M}^{+}, 35.45\right), 389$ (34.45), 275 (23.28), 178 (100), 145 (17.7), 95 (22.3).

\subsection{Microbiological Procedures for the Activity Study}

\subsubsection{Materials and method}

Media: Nutrient agar and Potato Dextrose Agar[6,7] plates were used for bacterial and fungal organisms respectively.

\subsubsection{Preparation of microbial suspension}

The bacterial and fungal strains were subculture at $37^{\circ} \mathrm{C}$ for six hrs in the corresponding medium of three successive days. These suspensions were used to insulate the antibiograms.

\subsubsection{Preparation of the biograms}

The agar disk diffusion method was performed on each of the tested substance solution in dimethylformamide. Filter paper discs were impregnated with $1 \mathrm{ml}$ of the solution and placed on the inoculated plates. These plates after standing at $4^{\circ} \mathrm{C}$ for 2 hours were incubated at $37^{\circ} \mathrm{C}$ for 24 hours. The diameters of the inhibition zones were measured in millimeters.

\section{Conclusions}

A series of novel substituted quinazolone derivatives were synthesized by the reaction of benzoxazinone derivative $\mathbf{2}$ with some primary aromatic amines. All the compounds were subjected to biological screening and they showed promising antibacterial and antifungal activity which were 
comparable to the activity of known standard drugs where compounds $3,5,7,8,9,10,11,17$ and 18 exhibited good activities against the reference chemotherapeutics due to the presence of antipyrine, pyridine and phthalimide moieties which play an important as antibacterial and antifungal, while 8, 9, 10 and 18 exhibited good activities against $B a$ cillus Thuringenesis and 5, 10, 11, 14, 15, 17 and 18 exhibited good activities against Klebseilla Pneumonia. On the other hand, the results for antifungal activities revealed that, compounds 5, 6, 7, 16 and 17 exhibited good activities against Trichoderma Herzianum and Trichoderma Virdi. This proves the high therapeutic value of these compounds and encourages further study to explore their biological potential.

\section{REFERENCES}

[1] K. Waisser, E. Petrlíková, M. Peřina, V. Klimešová, J. Kuneš, K. Palát, J. Kaustová, H. Dahse, U. Möllmann, European Journal of Medicinal Chemistry, 45, 2719-2725, 2010.

[2] X. Li, N. Liu, H. Zhang, S. E. Knudson, R. A. Slayden, P. J. Tonge, Bioorganic \& Medicinal Chemistry Letters, 20, 6306-6309, 2010.

[3] D. Zhou, L. B. Harrison, U. Shah, H. T. Andree, A. G. Hornby, R. Scerni, E. L. Schechter, L. D. Smith, M. K. Sullivan, E. R. Mewshaw, Bioorg. Med. Chem . Lett. 16, 1338, 2006.

[4] O. M. O. Habib, E. B. Moawad, S. D. Badawy, J. A. F. Mansour, J. Prakt. Chem. 332, 791, 1990.

[5] D. J. Wesley, H. E. Henry, J. Am. Chem. Soc. 78, 2543, 1956.

[6] A. A. El-Khamry, S. El-Nagdy, E. M. Shaban, Egypt J. Chem. 31, 241, 1988.

[7] S. R. Verma, L. W. Nobles, J. Pharm. Sci. 57, 1801, 1968. 